\title{
REHIDRATACIÓN POST-EJERCICIO CON AGUA DE COCO: ¿IGUAL O MÁS EFECTIVA QUE UNA BEBIDA DEPORTIVA?
}

\author{
Alexandra Pérez Idárraga y Luis Fernando Aragón Vargas \\ Escuela de Educación Física y Deportes, Universidad de Costa Rica, \\ alpida11@gmail.com
}

\begin{abstract}
RESUMEN
Objetivo: comparar la efectividad rehidratante del agua de coco fresca, con el agua embotellada y una bebida deportiva. Metodología: once participantes de 22,0 $\pm 1,9$ años y $65,6 \pm 13,0 \mathrm{~kg}$ de masa corporal (promedio \pm D.E), asistieron al laboratorio en tres ocasiones, separadas entre sí por una semana. En cada sesión se deshidrataron por ejercicio en el calor hasta perder $1,84 \pm 0,2 \%$ de la masa corporal. Luego fueron rehidratados en una hora, con agua comercial embotellada (AE), una bebida deportiva (BD) o agua de coco (AC), utilizando un volumen equivalente al $120 \%$ del peso perdido. Se evaluaron las sensaciones percibidas relacionadas con la tolerancia y la aceptación de las bebidas. Se recolectó la orina eliminada durante tres horas post-rehidratación. Resultados: Hubo interacción entre las bebidas y el tiempo sobre la orina excretada $(p=0,003)$, siendo el AE estadísticamente diferente al AC y la BD a los 30 y 60 min post-rehidratación $(p<0,05)$. El volumen total de orina fue mayor con agua embotellada $(625 \pm 183 \mathrm{~mL})$, en comparación con AC y BD $(390 \pm 73 \mathrm{~mL}$ y $416 \pm 200 \mathrm{~mL}$, respectivamente, $p<0,002)$; AC y BD no fueron distintas entre sí $(\mathrm{p}>0,05)$. Esto produjo porcentajes de conservación de líquido diferentes para $\mathrm{AE}(56 \%)$, con respecto a $\mathrm{BD}(71 \%)$ y $\mathrm{AC}(71 \%)(p<0,001)$. No hubo interacción entre las bebidas y el tiempo sobre las sensaciones percibidas de tolerancia $(p>0,05)$. La percepción de la dulzura fue menor para $\mathrm{AE}(p=0,024)$. BD obtuvo mejores puntajes de sabor y aceptación general $(p<0,05)$. Conclusiones: el agua de coco fresca resulta tan efectiva como una bebida deportiva para conservar el líquido consumido, teniendo una buena tolerancia y aceptación. Podría ser usada como una bebida rehidratante, cuando la persona la tenga disponible y le agrade su sabor.
\end{abstract}

PALABRAS CLAVES: eliminación de la orina, recuperación, hidratación, deshidratación, ejercicio.

\section{POST-EXERCISE REHYDRATION WITH COCONUT WATER: AS EFFECTIVE AS A SPORTS DRINK, OR BETTER?}

\begin{abstract}
Objective: To compare the effectiveness of fresh coconut water as a rehydration beverage with bottled water and a sports drink. Method: eleven participants, $22.0 \pm 1.9$ years of age and $65.6 \pm 13.0 \mathrm{~kg}$ body mass (average \pm S.D.), reported to the laboratory on three separate occasions, each one week apart. Each time they exercised in the heat until they were dehydrated by $1.84 \pm 0.2 \%$ of initial body mass. They were then rehydrated for one hour with commercial bottled water (BW), a sports drink (SD), or coconut water (CW), using a volume equivalent to $120 \%$ of weight loss. Perceived beverage acceptance and tolerance ratings were evaluated, and urine was collected over three hours following rehydration. Results: Urine volumes showed an interaction between beverage and time $(p=0.003)$; BW was statistically different from CW and SD, 30 and 60 minutes after rehydration $(p<0.05)$. Total urine volume was higher for BW $(625 \pm 183 \mathrm{~mL})$ compared with both $\mathrm{CW}$ and $\mathrm{SD}(390 \pm 73 \mathrm{~mL}$ and $416 \pm 200 \mathrm{~mL}$, respectively, $p<0.002)$; CW and $\mathrm{SD}$ were not different from each other $(\mathrm{p}>0.05)$. This resulted in a different fluid conservation percentage for BW (56\%) compared with CW $(71 \%)$ and SD $(71 \%)(p<0.001)$. Perceived tolerance ratings showed no interaction between beverage and time $(p>0.05)$. Perceived sweetness was lower for BW $(p=0.024)$; SD had higher scores for flavor and overall acceptance $(p<0.05)$. Conclusions: fresh coconut water is as effective as a sports drink in terms of fluid retained following consumption, and it has good tolerance and acceptance; it could be used for rehydration provided it is accessible to the individual and the person finds the flavor pleasing.
\end{abstract}

KEY WORDS: urine elimination, recovery, hydration, dehydration, exercise. 


\section{MHSalud}

URL www.una.ac.cr/mhsalud

\section{INTRODUCCIÓN}

El agua de coco ha sido una bebida de gran interés en el campo investigativo, desde hace muchos años. En algunos estudios publicados durante la época de la II Guerra Mundial o poco después de ésta, se resaltó su alto valor biológico, su uso médico para la alimentación de los niños y la posibilidad de administración por la vía oral o intravenosa, para efectuar la rehidratación, demostrando una baja incidencia de reacciones alérgicas y, en general, de efectos adversos (SotoPradera, Fernández \& Calderin, 1942; Eiseman, 1954).

Más adelante, algunas investigaciones demostraron su utilidad como un rehidratante por vía oral o intravenosa, durante algunas epidemias de cólera o en casos de emergencia (Kuberski, Roberts, Linehan, Bryden, \& Teburae, 1979; Campbell-Falck, Thomas, Falck, Tutuo, \& Clem, 2000), e incluso en el post operatorio de cirugías relacionadas con el tracto digestivo (Iqbal, 1976). En cualquiera de los casos mencionados, los autores han sido enfáticos al señalar que el agua de coco no debería ser usada como un sustituto de las soluciones de rehidratación oral o intravenosa, como las recomendadas actualmente por la Organización Mundial de la Salud (World Health Organization, 2006).

Las investigaciones en el área han llevado a concluir que la composición del agua de coco, al igual que la mayoría de los productos naturales, cambia de acuerdo con su estado de maduración, la zona, la región y el tipo de palma (Child \& Nathanael, 1950). Con todo y su variación, se ha visto que generalmente el agua de coco presenta un alto contenido de potasio, calcio y magnesio y un bajo contenido de sodio, en comparación con el plasma humano. Su contenido de carbohidratos (glucosa, fructosa e inulina) puede oscilar entre 1,5 y 5,5 g/100 mL (Kuberski et al., 1979; Vigliar, Sdepanian \& Fagundes-Neto, 2006).

Los estudios más recientes con agua de coco no se han enfocado a su uso clínico, como en épocas pasadas, sino a su utilidad como rehidratante oral después del ejercicio (Aragón-Vargas \& Madriz-Dávila, 2000; Saat, Singh, Sirisinghe \& Nawawi, 2002), incluso, algunos la han estudiado con adición de sodio, procurando mejorar su efectividad (Ismail, Singh \& Sirisinghe, 2007). Con algunas discrepancias entre sí, en estos estudios se apoya su uso como bebida en la rehidratación post-ejercicio.

Si se tiene en cuenta que el objetivo de la rehidratación post-ejercicio consiste en aprovechar al máximo el líquido consumido, reduciendo la diuresis en la mayor medida posible y logrando de este modo una mayor conservación de la bebida consumida, se puede afirmar que el agua de coco en condiciones de ejercicio tiende a ser superior al agua; sin embargo, no muestra ni superioridad ni desventajas claras frente a las bebidas deportivas.

Se sabe que las bebidas deportivas, debido a su composición con electrolitos y carbohidratos, pueden favorecer una mayor conservación de líquido y por eso han sido el patrón de comparación frente a otras bebidas, las cuales han surgido como alternativas de rehidratación, mientras que el agua, la bebida por excelencia, tiende a incrementar la eliminación de la orina y a disminuir la sensación de sed, antes de lograr una completa hidratación (Costill \& Sparks, 1973; GonzálezAlonso, Heaps \& Coyle, 1992; Shirreffs \& Maughan, 1998).

Dadas las ventajas potenciales del agua de coco como una bebida deportiva y la alta disponibilidad en las diferentes zonas, ha aumentado el interés de la Organización de las Naciones Unidas para la Agricultura y la Alimentación (FAO, Food and Agriculture Organization) por mejorar su manipulación y conservación, promoviendo su consumo, del tal 
manera que adquirió una patente para el proceso del embotellado, el cual pretende proteger las propiedades microbiológicas y organolépticas del agua de coco sin la adición de conservantes, para que pueda ser usada a pequeña y mediana escala (Rolle, 2007).

Ya que la composición del agua de coco consumida en Costa Rica podría ser suficientemente distinta de la estudiada en otras latitudes, por las razones anteriormente citadas, el objetivo del presente estudio fue comparar la efectividad del agua de coco costarricense procesada y embotellada según los lineamientos de la FAO, con agua comercial embotellada y con una bebida deportiva convencional, para la rehidratación post-ejercicio. Se evaluó tanto la conservación de líquido en el cuerpo, como su aceptación y tolerancia.

\section{METODOLOGÍA}

Participantes. Once participantes voluntarios sanos (seis hombres y cinco mujeres), físicamente activos (actividad física al menos, tres veces por semana, una hora por sesión), de 22,0 $\pm 1,9$ años de edad y $65,6 \pm 13,0 \mathrm{~kg}$ de peso $(\bar{x} \pm$ D.E), firmaron un consentimiento informado, previamente aprobado por el Comité Ético Científico de la Universidad. Llenaron un cuestionario con la información de salud pertinente y recibieron las indicaciones por escrito para su participación.

Instrumentos. El ejercicio orientado al logro de la deshidratación se realizó dentro de un cuarto de ambiente controlado, $\left(32^{\circ} \mathrm{C}\right.$ y $50-60 \%$ de humedad relativa). Los participantes realizaron ejercicio en una banda rodante Sports Art 3250 y en Cicloergómetros Monark ${ }^{\circledR} 818 \mathrm{c}$. Se controló la intensidad del ejercicio con un monitor de frecuencia cardíaca Polar ${ }^{\circledR}$ S120: los participantes debían mantener una intensidad moderada-alta del ejercicio, equivalente al $80-85 \%$ de la frecuencia cardíaca máxima, calculada según la fórmula: 207-(0,7 x edad en años) propuesta por Gellish et al. (2007). Para tomar el peso de los participantes, se utilizó una Báscula Ballar ${ }^{\circledR}$ Modelo DSB921, con una capacidad máxima de $250 \mathrm{~kg}$ y una sensibilidad de $10 \mathrm{~g}$.

La orina fue recolectada en recipientes de plástico debidamente marcados para cada participante. Tanto la bebida suministrada como la orina fueron pesadas en una báscula marca DHAUS ${ }^{\circledR}$ Modelo CS-2000, con una capacidad de $2000 \mathrm{~g}$ y sensibilidad de $1 \mathrm{~g}$. Adicionalmente, se midió la gravedad específica de la orina (USG, por su sigla en inglés), con un refractómetro Hand-Held $A T A G O^{\circledast,}$ modelo URC-NE con una escala de 1,000 hasta 1,050. Se fijó el estado de euhidratación con una USG $\leq 1,020$ (Sawka et al. 2007). Para analizar las sensaciones percibidas de tolerancia (sed, náuseas, dolor abdominal, llenura y urgencia de defecación), se utilizó una escala entre 1 (ausencia de sensación) hasta 5 (mucha sensación), similar a la utilizada por Morton, Aragón Vargas \& Callister (2004). Se usó una escala similar con el fin de dar un puntaje de sabor, dulzura y aceptación general de la bebida, similar a las empleadas en estudios previos con agua de coco (Saat et al., 2002; Ismail et al., 2007).

Procedimientos. Los participantes recibieron indicaciones de preparación para cada sesión. Ellos debían registrar todos los alimentos y bebidas cada día, previo a su asistencia al laboratorio. Se les sugirió consumir alimentos similares durante esos días y tomar cinco vasos de agua extra, evitando el consumo de bebidas alcohólicas, cafeinadas (energizantes, café, té, gaseosas) o muy dulces, diuréticos, laxantes o antihistamínicos. Se les solicitó abstenerse de practicar ejercicio extenuante veinticuatro horas antes de cada sesión. 


\section{MHSalud \\ URL www.una.ac.cr/mhsalud}

Éste es un estudio experimental de medidas repetidas. Cada participante asistió al laboratorio en tres ocasiones diferentes, separadas entre sí por una semana; en cada sesión ingirió una bebida distinta. El orden de las bebidas fue asignado en forma aleatoria.

Los participantes llegaron en ayunas al laboratorio (mínimo ocho horas de ayuno). Se recolectó una muestra de orina a la cual se le midió la gravedad específica, para obtener una idea aproximada del estado de hidratación en el cual llegaban al estudio. Se les suministró un desayuno estandarizado, que aportó $775 \pm 25 \mathrm{Kcal}$ (distribuidas en $73 \%$ carbohidratos, $17 \%$ grasas, y $10 \%$ proteínas); $1300 \mathrm{mg} \mathrm{Na}^{+}$y $350 \mathrm{~mL}$ de líquido. Este desayuno fue el único alimento que recibieron hasta el final del experimento. Después de consumirlo esperaron aproximadamente una hora antes de iniciar el ejercicio, momento en el cual se pesaron desnudos y secos (Peso Inicial).

La deshidratación se logró mediante el ejercicio intermitente en el calor, alternando entre banda rodante y cicloergómetro (diez minutos en cada máquina) y descansando cada veinte minutos durante cinco minutos, momento en el cual eran pesados desnudos y secos. El ejercicio continuó hasta que cada participante alcanzó una deshidratación cercana al 2\% de la masa corporal (MC), usando como referencia el Peso Inicial. Para continuar, los participantes se ducharon y evacuaron completamente la vejiga y después se pesaron (Peso Post-ejercicio).

Se calculó el volumen total del líquido que cada persona debería consumir para su rehidratación, a saber, el equivalente en $\mathrm{mL}$ a $120 \%$ del Peso Perdido durante el ejercicio (que es el Peso Inicial menos el Peso Post-ejercicio). El volumen total se dividió en cuatro porciones iguales y cada porción se ingirió cada quince minutos.

Las bebidas utilizadas (Tabla 1) fueron agua comercial embotellada marca Cristal $^{\circledR}$ (AE), bebida deportiva marca Gatorade ${ }^{\circledR}$ (BD) y agua de coco (AC) extraída semanalmente de unos veinticinco a treinta cocos, que luego fue embotellada y refrigerada siguiendo las recomendaciones de la FAO (Rolle, 2007).

Tabla 1.

Composición de las bebidas utilizadas para la rehidratación

\begin{tabular}{lccc}
\hline \multicolumn{1}{c}{ Componente } & Agua & $\begin{array}{c}\text { Agua de } \\
\text { Coco* }\end{array}$ & $\begin{array}{c}\text { Bebida } \\
\text { Deportiva }\end{array}$ \\
\hline $\begin{array}{l}\text { Sodio }(\mathbf{m E q} / \mathbf{L}) \\
\text { Potasio }(\mathbf{m E q} / \mathbf{L})\end{array}$ & 0,3 & $2,5 \pm 0,2$ & 18,0 \\
$\begin{array}{l}\text { Carbohidratos } \\
(\boldsymbol{\%})\end{array}$ & 0,0 & $51,0 \pm 2,0$ & $3,0 \pm 0,2$ \\
\hline
\end{tabular}

*El agua de coco fue analizada semanalmente, puesto que cada semana se embotellaron mezclas de cocos diferentes. Se reporta el promedio \pm desviación estándar. Los valores del agua y la bebida deportiva se reportan según la información de las etiquetas respectivas.

Antes de iniciar el proceso de la rehidratación y cuatro veces durante la misma, los participantes puntuaron en una escala las sensaciones percibidas de tolerancia y sed en cada bebida (cinco mediciones por variable); los valores de agrado se promediaron para obtener un único puntaje por persona por bebida para cada variable: dulzura, sabor, y aceptación general. Antes de iniciar el 


\section{MHSalud}

URL www.una.ac.cr/mhsalud

estudio, y por una única vez, ellos evaluaron también la importancia que le daban a ciertas características de una bebida para la rehidratación después del ejercicio.

Al finalizar el período de rehidratación se solicitó a los participantes vaciar completamente la vejiga, recolectando la orina en recipientes plásticos debidamente pesados y marcados. A partir de ese momento, vaciaron de nuevo totalmente la vejiga cada treinta minutos, durante tres horas, para un total de siete volúmenes parciales de orina. Cada recipiente con orina se pesó al gramo más cercano (para calcular el volumen) y se desechó.

La masa corporal (en kg, hasta el decagramo más cercano) de los participantes se midió durante el estudio: al inicio (Peso Inicial), después del ejercicio (Peso Post-ejercicio), después de la rehidratación (Peso post-rehidratación) y al final de la recolección de las muestras de orina (Peso Final).

Para calcular el balance neto de fluidos (BNF), se tomó el valor del peso inicial como estado de euhidratación (es decir, cero), y luego se consideraron los cambios en la masa corporal en los distintos momentos (i). Los valores obtenidos contemplan no solamente las pérdidas por sudoración y las ganancias por ingesta de líquido para la rehidratación, sino también las pérdidas del líquido a través de la orina, el sudor y la respiración. Estas últimas dos no fueron medidas directamente. Todos los valores de la fórmula están dados en mililitros $(\mathrm{mL})$, asumiendo una equivalencia de un mililitro por gramo.

$$
B N F i=\text { Peso }_{i}-\text { Peso Inicial }
$$

Adicionalmente, se calculó el porcentaje de conservación de líquido para cada bebida, según la fórmula:

$$
\% \text { ConservaciónLíquido }=\frac{\text { LíquidoIngerido }- \text { Vol.Orina }}{\text { LíquidoIngerido }} * 100
$$

Este porcentaje representa cuánto del líquido consumido después del ejercicio no fue eliminado, a través de la orina, durante las tres horas posteriores a la rehidratación.

Análisis de los datos. Los datos se procesaron en el paquete estadístico SPSS, versión 15.0 para Windows. Se obtuvo la estadística descriptiva (promedio \pm D.E) para caracterizar la muestra y se verificó la distribución normal de cada variable con la prueba de Shapiro-Wilk. Se realizaron análisis de varianza (ANOVA) de una vía (factor bebidas), para cada una de las siguientes variables: peso inicial, USG inicial, tiempo de ejercicio, deshidratación post-ejercicio y volumen de líquido consumido, con el fin de verificar que las condiciones previas a la rehidratación o durante ésta fueran iguales para las tres bebidas. Luego, se aplicó un ANOVA de dos vías de medidas repetidas para la orina eliminada en el tiempo ( 3 bebidas X 7 mediciones). Se realizaron ANOVA de una vía de medidas repetidas (tres bebidas), para obtener el porcentaje de la conservación de líquido y para el volumen total de la orina. Se hicieron ANOVA de dos vías de medidas repetidas, con el balance neto de fluidos (3 bebidas X 4 mediciones) y con cada una de las diferentes sensaciones percibidas ( 3 bebidas $\mathrm{X} 5$ mediciones).

Para las variables relacionadas con el agrado de las tres bebidas, se realizaron ANOVA con una vía de medidas repetidas (tres bebidas) para la dulzura, el sabor, y la aceptación general. Cuando se identificaron efectos principales, se efectuó una evaluación post hoc mediante la prueba de 


\section{MHSalud}

URL www.una.ac.cr/mhsalud

Bonferroni, con el fin de identificar dónde estaba la diferencia. Como información complementaria, se evalúo también la importancia que los participantes daban a algunas características de la bebida seleccionadas arbitrariamente, calculando el promedio de la puntuación para cada característica.

\section{RESULTADOS}

El protocolo de deshidratación tuvo una duración de 55,3 \pm 8,9 minutos (promedio \pm desviación estándar) de ejercicio intermitente en el calor; los participantes perdieron 1,2 $\pm 0,3 \mathrm{~kg}$ de la masa corporal $(\mathrm{MC})$, sin diferencias entre las tres condiciones experimentales $(\mathrm{p}=0,156)$. Esto representó una deshidratación de 1,84 $\pm 0,2 \%$ MC. Tampoco hubo diferencias en las condiciones previas a la rehidratación, ni en el volumen del líquido ingerido para la rehidratación (1464 \pm 110 $\mathrm{mL}$ ) (Ver la Tabla 2).

Tabla 2.

Condiciones iniciales

\begin{tabular}{|c|c|c|c|c|c|c|}
\hline \multirow[t]{2}{*}{ MEDICIÓN } & \multicolumn{2}{|c|}{$\begin{array}{c}\text { AGUA } \\
\text { EMBOTELLADA }\end{array}$} & \multicolumn{2}{|c|}{$\begin{array}{c}\text { BEBIDA } \\
\text { DEPORTIVA }\end{array}$} & \multicolumn{2}{|c|}{ AGUA DE COCO } \\
\hline & $x$ & D.E & $x$ & D.E & $x$ & D.E \\
\hline USG inicial $(\mathrm{g} / \mathrm{mL})$ & 1,022 & 0,003 & 1,013 & 0,003 & 1,017 & 0,005 \\
\hline Peso inicial (kg) & 65,66 & 13,15 & 65,79 & 12,86 & 65,49 & 13,03 \\
\hline Tiempo de ejercicio (min) & 54,5 & 9,3 & 55,5 & 9,3 & 56,0 & 8,0 \\
\hline Deshidratación (\% MC) & 1,84 & 0,21 & 1,91 & 0,24 & 1,79 & 0,22 \\
\hline Volumen para rehidratación $(\mathrm{mL})$ & 1450 & 334 & 1520 & 388 & 1420 & 412 \\
\hline
\end{tabular}

En la Figura 1 se puede observar el comportamiento del volumen de orina correspondiente a cada una de las tres bebidas. Hubo interacción significativa entre las bebidas y el tiempo $(\mathrm{p}=0,003)$, siendo el AE estadísticamente diferente al AC y la BD a los 30 y 60 min post-rehidratación $(\mathrm{p}<0,05)$. 


\section{MHSalud}

URL www.una.ac.cr/mhsalud

Figura 1. Volúmenes parciales de orina eliminada durante la prueba.

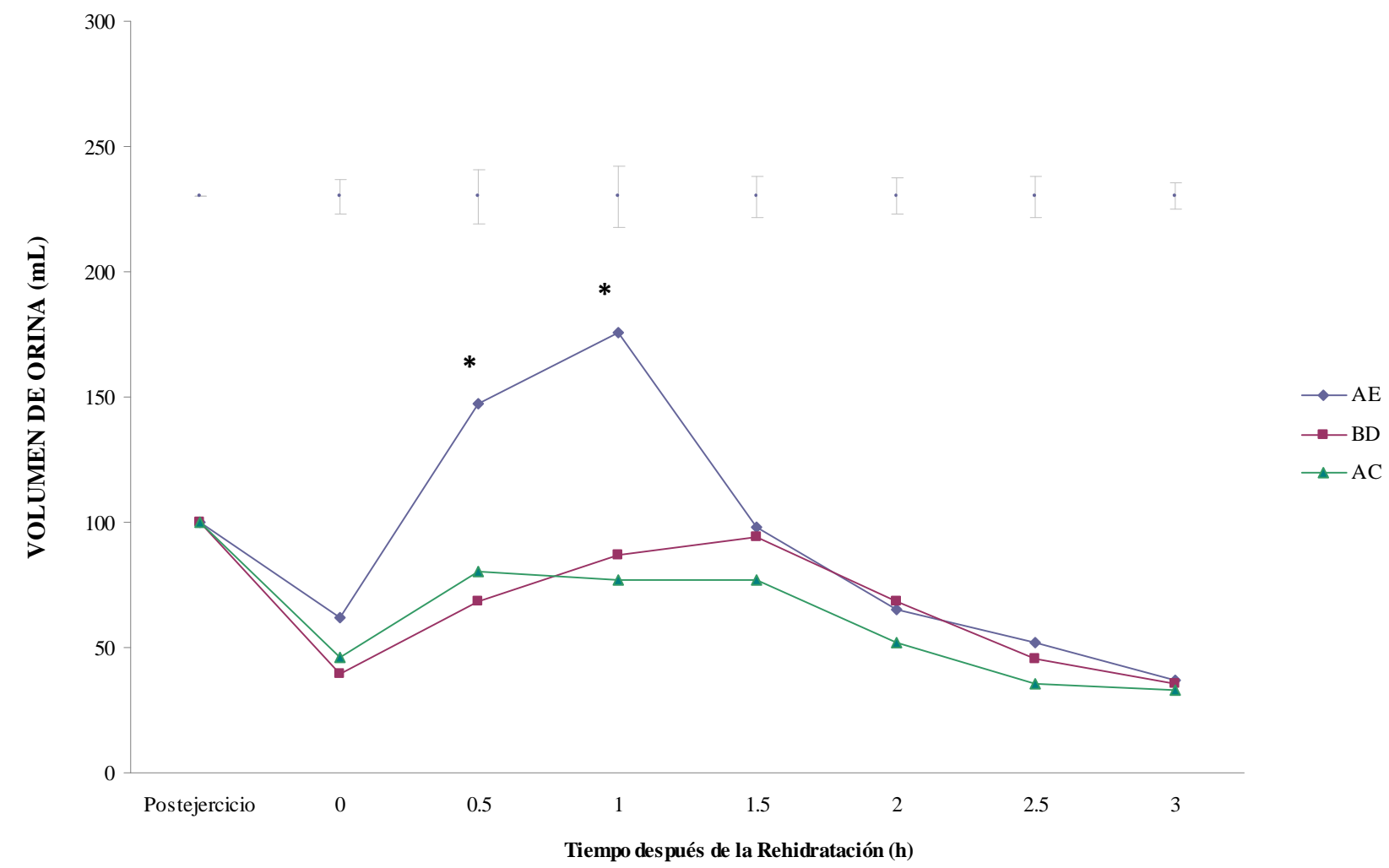

Figura 1. (*) p <0,05, AE vs AC y BD. Las barras superiores corresponden el error típico grupal de la medición. La rehidratación ocurrió entre el postejercicio y el tiempo 0. AE agua embotellada, BD bebida deportiva, AC agua de coco.

La Figura 2 muestra que el volumen total de la orina fue mayor con AE $(625 \pm 183, p=0,002)$ que con $\mathrm{AC}(390 \pm 73 \mathrm{~mL})$ o $\mathrm{BD}(416 \pm 200 \mathrm{~mL})$; sin embargo, estas últimas no fueron distintas entre sí $(p>0,05)$. También se puede observar en la Figura 2 que el porcentaje de conservación de líquido fue menor con $\mathrm{AE}(55,95 \pm 13,48 \%, \mathrm{p}<0,05)$, que con las otras dos bebidas (BD 71,29 \pm $14,26 \%$ y AC $71,05 \pm 7,90 \%)$. 


\section{MHSalud}

URL www.una.ac.cr/mhsalud

Figura 2. Porcentaje de conservación de líquido con cada bebida.

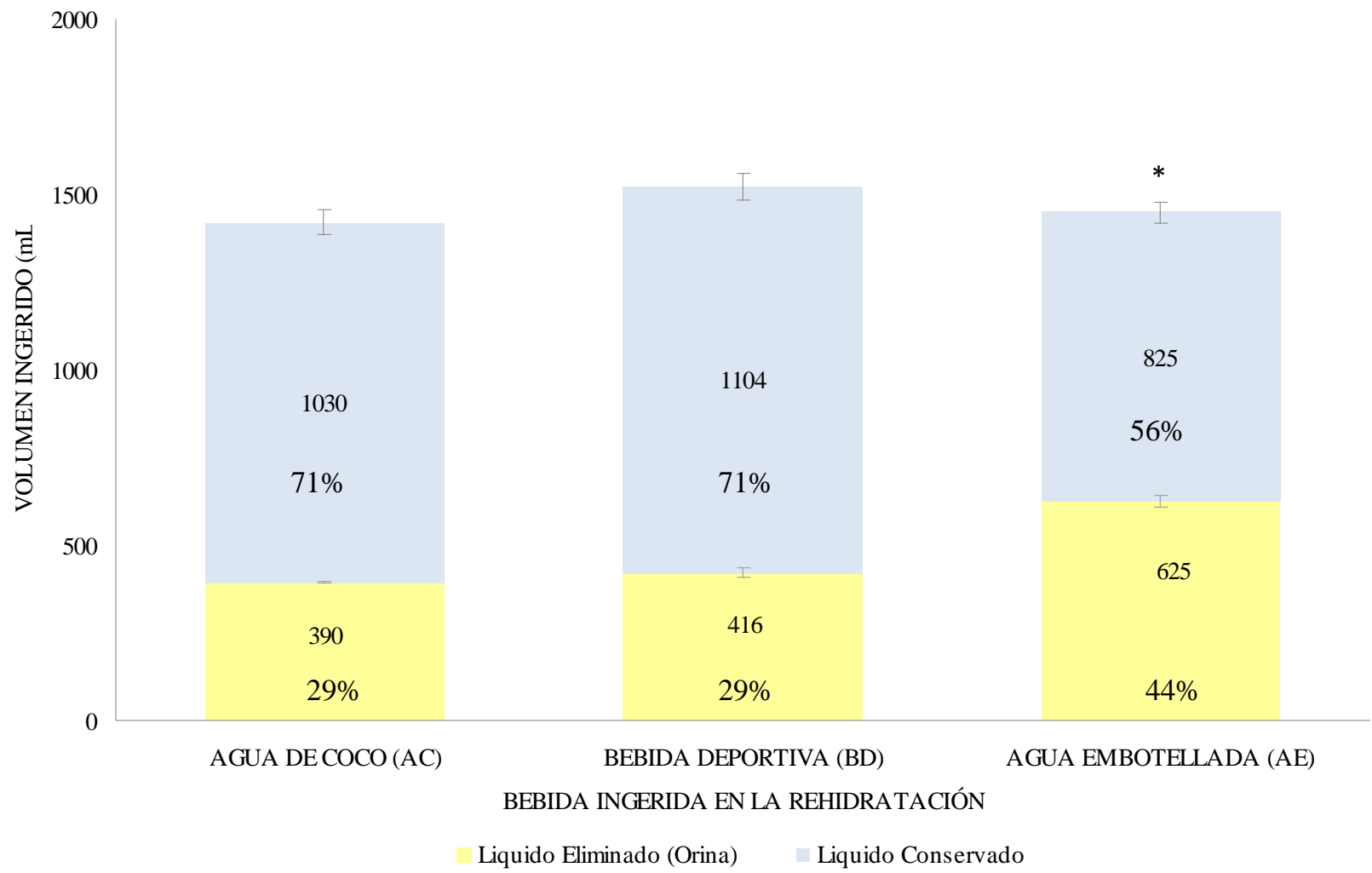

Figura 2. Comparación de los volúmenes totales de orina, $\mathrm{p}=0,002$; comparación del porcentaje del líquido conservado, $\mathrm{p}=0,001 ;(*) \mathrm{AE}$ distinto de AC y BD $(\mathrm{p}<0,05)$. Las barras corresponden al error típico. Los valores porcentuales se calcularon usando el volumen ingerido de cada bebida como valor de referencia (100\%). AE agua embotellada, BD bebida deportiva, AC agua de coco.

La Figura 3 muestra el balance neto de fluidos. Los participantes estuvieron en un balance negativo después de seguir el protocolo de la deshidratación, luego en la rehidratación se produjo un balance positivo breve, no obstante, al final de todo el período los participantes se encontraban en un balance negativo de líquidos, que fue significativamente mayor con $\mathrm{AE}(-580 \pm 110 \mathrm{~mL}$; $p=0,03)$ comparado con AC y BD $(-440 \pm 130 \mathrm{~mL}$ y $-420 \pm 160 \mathrm{~mL}$, respectivamente). 


\section{MHSalud}

URL www.una.ac.cr/mhsalud

Figura 3. Balance neto de fluidos en cada tiempo de medición, para cada bebida.

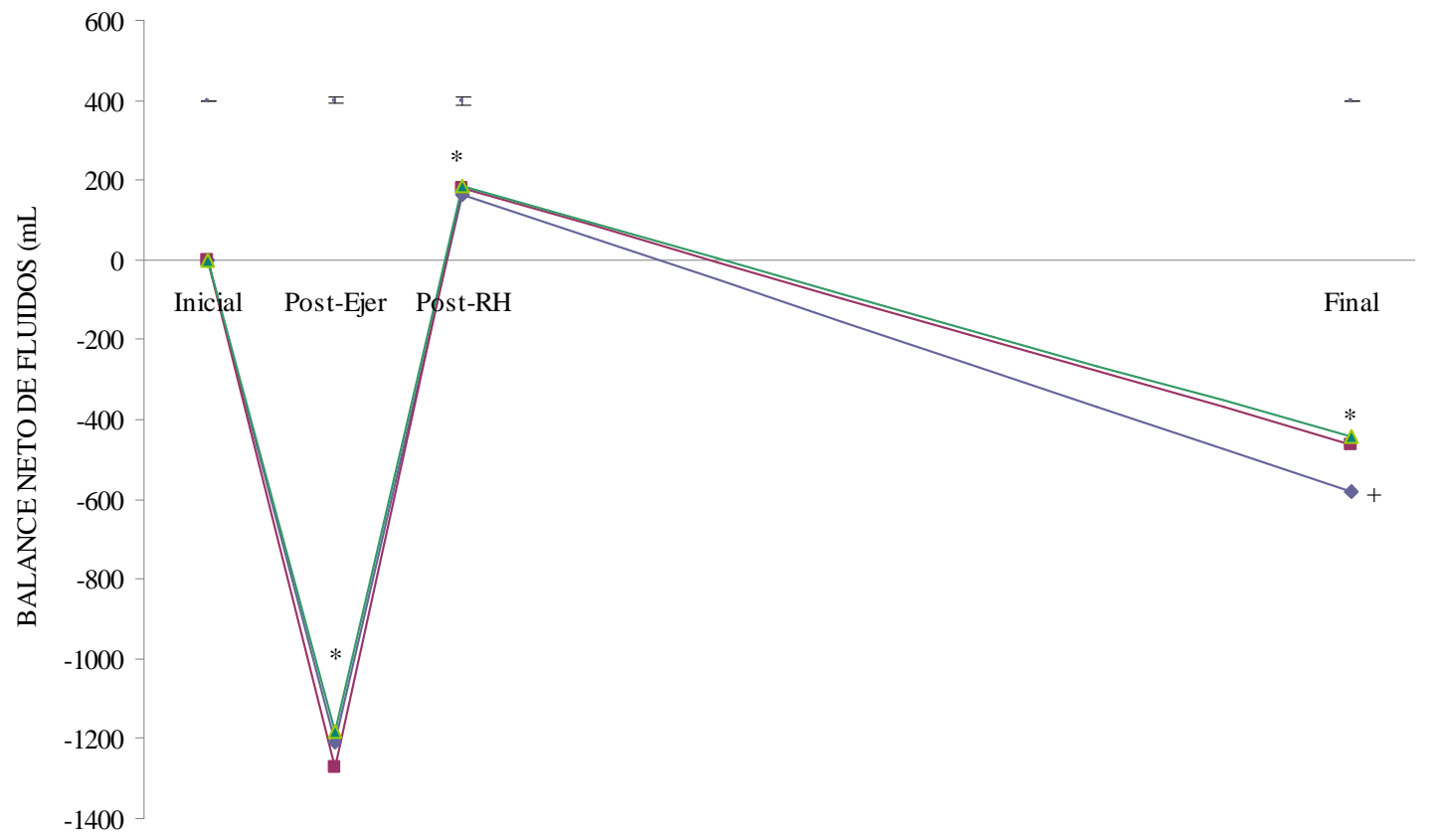

Figura 3. Interacción entre protocolo y tiempo: $p=0,011$ Efecto principal de bebida: $p=0,373$. Efecto de tiempo: $(*) p$ $<0,001$ vs. Peso inicial. (+) $p=<0,005$ vs. BD y AC. Las barras superiores representan el error típico grupal de la medición. AE agua embotellada, BD bebida deportiva, AC agua de coco.

En lo concerniente a las sensaciones percibidas de tolerancia, las Figuras $4 a-4 e$ muestran que no hubo interacción entre las bebidas y el tiempo $(p>0,05)$. Ninguna bebida produjo síntomas gastrointestinales medibles. A su vez, las tres bebidas disminuyeron por igual la sed e incrementaron simultáneamente la sensación de llenura, sin diferencias entre ellas. En general, las tres bebidas comenzaron a disminuir la sed después de los treinta minutos, de ahí en adelante, la percepción de sed fue menor a la reportada antes de iniciar la rehidratación $(p<0,05)$. La percepción de llenura en general para las tres bebidas fue mayor al final de la última porción, con respecto a la percepción antes de la rehidratación $(\mathrm{p}<0,05)$. En lo que se refiere a la aceptación, el nivel de percepción de dulzura fue menor en el $\mathrm{AE}(p=0,024)$, en relación con el AC y la $\mathrm{BD}$, los cuales no fueron diferentes entre sí. BD tuvo mejores puntajes con respecto al sabor y a la aceptación general, al compararla con $\mathrm{AC}$ y $\mathrm{AE}$ ( $p<0,05$, figura $4 f)$. 


\section{MHSalud}

URL www.una.ac.cr/mhsalud

Figura 4. Sensaciones percibidas de tolerancia y aceptación antes de y durante la rehidratación.

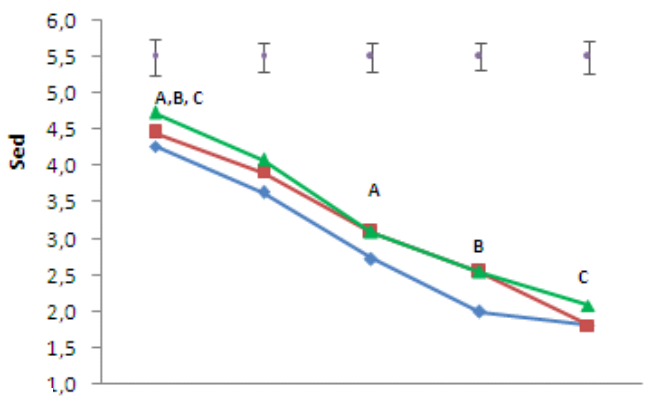

c)

e)
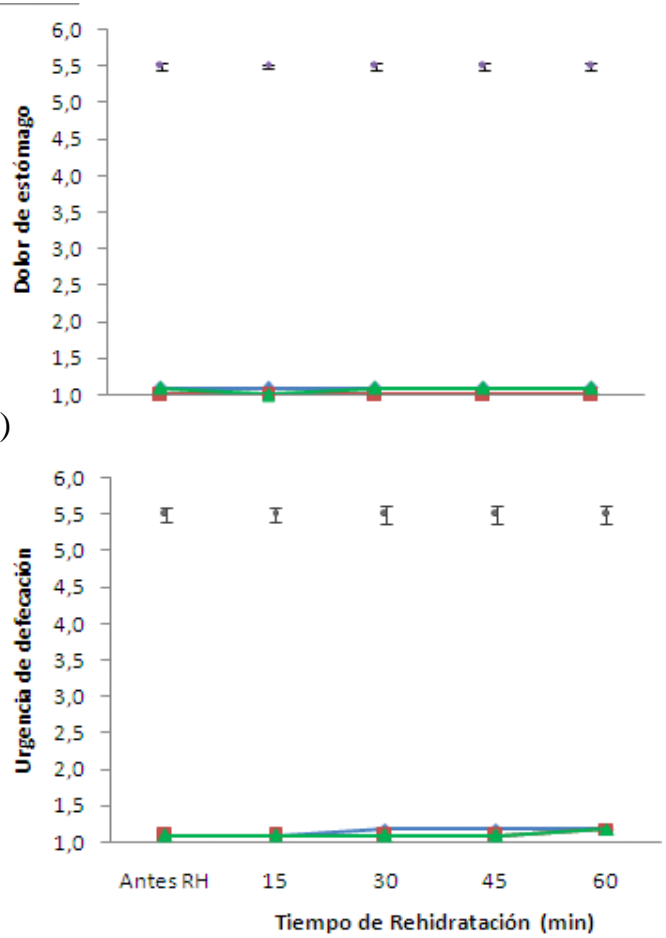

b)

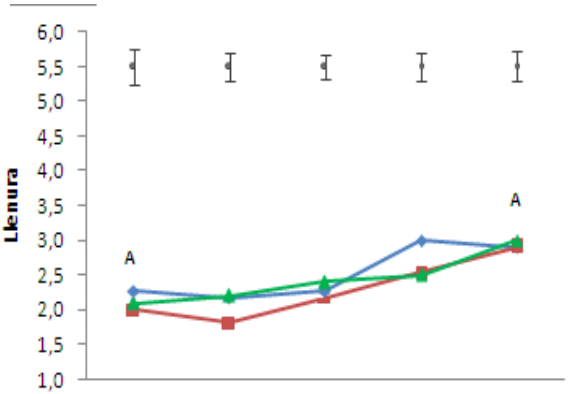

d)

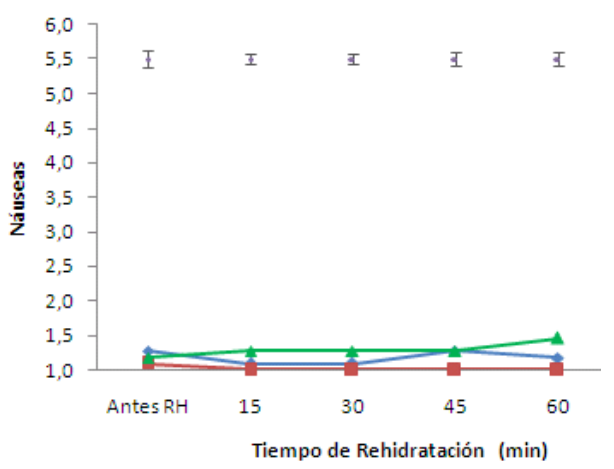

f)

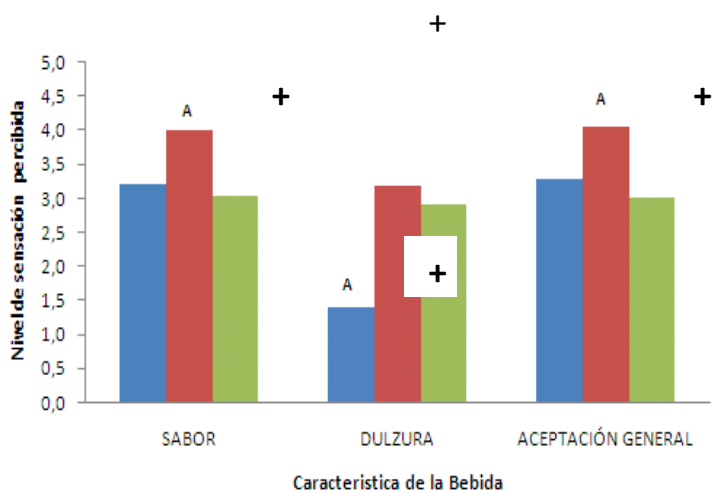

Figura 4. No se presentan interacciones entre la bebida y el tiempo $(p>0,05)$; las letras mayúsculas iguales indican $p<0,05$ entre mediciones, el símbolo (+) indica $p<0,05$ entre bebidas. Las barras superiores reflejan el error típico grupal de la medición. AE agua embotellada, BD bebida deportiva, AC agua de coco. Escalas subjetivas de 1 (mínimo) a 5 (máximo). 


\section{MHSalud}

URL www.una.ac.cr/mhsalud

Figura 5. Importancia relativa asignada a algunas características de una bebida para la rehidratación post-ejercicio. TGI: trastornos gastrointestinales.

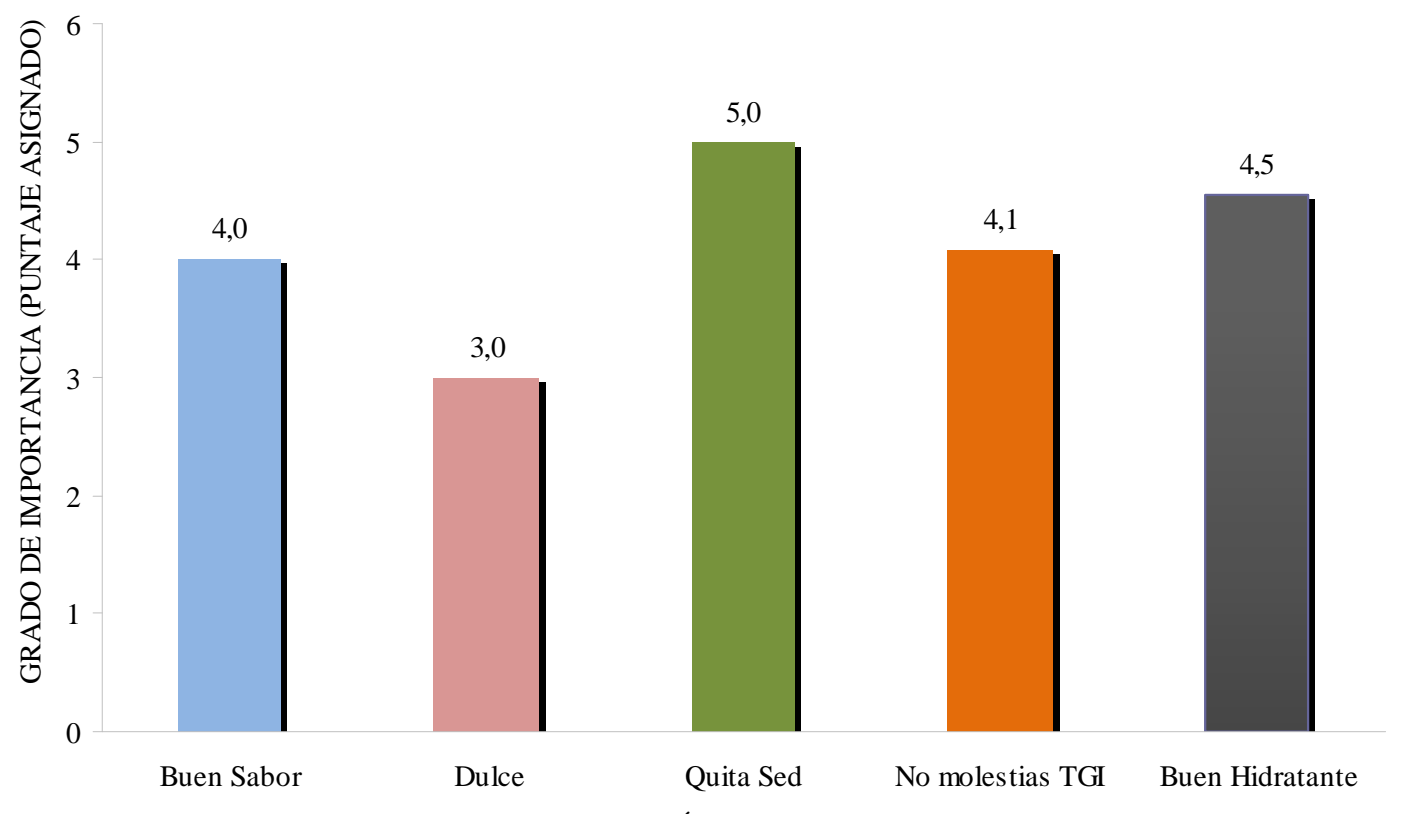

CARACTERÍSTICA DELA BEBIDA

La Figura 5 muestra la importancia relativa que le dieron los participantes del estudio a distintas cualidades de una bebida para la rehidratación después del ejercicio, resaltando que les quite la sed y que sea un buen hidratante; al atributo que le concedieron menor importancia fue al sabor dulce de la bebida.

\section{DISCUSIÓN}

En el presente estudio se evaluó la efectividad y la aceptación del agua de coco como bebida deportiva, así como la tolerancia a ella, al ser consumida después de una deshidratación equivalente al $2 \%$ de la masa corporal inducida por ejercicio. Los resultados sugieren que el agua de coco funciona tan bien como una bebida deportiva y mejor que el agua en la conservación del líquido que se consume durante la rehidratación. Sin embargo, la bebida deportiva obtuvo mayores puntajes con respecto al agrado del sabor y la aceptación general. El agua de coco y el agua no tuvieron diferencias en la aceptación, a pesar de ser el agua percibida como menos dulce. Todas las bebidas fueron bien toleradas en el nivel gastrointestinal, en los volúmenes utilizados (alrededor de 1,5 L en una hora).

En la etapa de la rehidratación se pretende que la bebida ingerida cumpla con diferentes requisitos, tales como estimular el consumo voluntario, evitar un incremento en la eliminación de la orina y lograr una pronta y efectiva recuperación de los fluidos perdidos a través del sudor. Todas las características las han cumplido hasta cierto punto algunas bebidas deportivas, debido a su contenido de electrolitos y carbohidratos que favorecen el proceso de la rehidratación 
(González-Alonso et al., 1992; Shirreffs, Armstrong \& Cheuvront, 2004; Casa, Clarkson \& Roberts, 2005; Sawka et al., 2007).

El agua durante la rehidratación no es tan efectiva, pues se sabe que al ser consumida en grandes volúmenes y corto tiempo, provoca un aumento sustancial en los volúmenes de orina eliminados (Shirreffs \& Maughan, 1997; Shafiee et al., 2005); esto es consistente con los resultados del presente estudio, donde el agua produjo volúmenes de orina significativamente, más altos.

Por el contrario, las bebidas que contienen electrolitos pueden resultar más efectivas durante la rehidratación después del ejercicio (Maughan, Owen, Shirreffs \& Leiper, 1994; Shirreffs et al., 2004), como ocurrió en este estudio con la bebida deportiva (atribuible a su contenido de sodio) y con el agua de coco (atribuible a su contenido de potasio, vide infra): éstas obtuvieron porcentajes de conservación iguales entre sí, aunque significativamente mejores que el agua.

En el análisis de las diferencias existentes en la efectividad de la rehidratación entre las bebidas, sería deseable comparar los porcentajes de conservación de líquido o los índices de rehidratación en diferentes estudios. Esto no es posible, debido a que los tiempos de recolección de la orina y los protocolos de la deshidratación y la rehidratación son a menudo distintos. Sin embargo, siempre se pueden comparar las cualidades del agua de coco con las de otras bebidas en cada estudio, intentando de este modo alcanzar una visión más clara sobre su utilidad como bebida para la rehidratación post-ejercicio. Una de las primeras investigaciones de rehidratación postejercicio con agua de coco fue un estudio de campo realizado por Madriz-Dávila (2000), en la provincia de Limón, una zona calurosa de Costa Rica. Ella detectó una diferencia en los niveles de reposición de líquido a favor del agua de coco $(27,6 \pm 16,0 \%)$ y la bebida deportiva $(28,1 \pm 19,0 \%)$ al compararlas con el agua $(11,8 \pm 25,0 \%, p<0,05)$. Utilizando este indicador, concluyó que el agua de coco es una bebida que podría ser recomendada para la rehidratación después del ejercicio.

En un estudio posterior, Saat et al. (2002) no encontraron diferencias significativas entre el agua $(73 \pm 5 \%)$, el agua de coco $(75 \pm 5 \%)$ y una bebida deportiva $(80 \pm 4 \%)$, con respecto al líquido que fue conservado en el cuerpo, ni tampoco en las variables estudiadas relacionadas con el éxito de la rehidratación, como el cambio en el volumen sanguíneo, la orina acumulada y el balance neto de fluidos. Sin embargo, los autores concluyeron que el agua de coco se comportó mejor que la bebida deportiva y el agua, al producir menos náuseas, llenura y dolor de estómago. En el presente estudio, no hubo diferencias en el nivel de tolerancia de las tres bebidas utilizadas, sin embargo, el volumen de bebida consumido fue menor y el protocolo de rehidratación diferente, con respecto al tiempo y la distribución del volumen.

Ismail et al. (2007), evaluaron no sólo las bebidas anteriores, sino que también incluyeron una cuarta bebida, el agua de coco con adición de sodio $\left(\mathrm{AC}-\mathrm{Na}^{+}\right)$. En las conclusiones del estudio, los autores sugieren que la adición de sodio al agua de coco natural podría conducir a resultados similares a la bebida deportiva, con una mejor tolerancia. Sin embargo, la estadística y las figuras presentadas en la publicación con respecto al porcentaje de líquido conservado, muestran que el agua es la única bebida significativamente diferente $(58,9 \pm 9,0 \%, \mathrm{p}<0,05)$, mientras que las demás

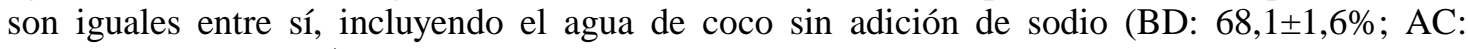
$65,1 \pm 1,7 \%$ y AC-Na ${ }^{+}: 69,4 \pm 1,4 \%$ ). Dicho resultado va de acuerdo con lo encontrado en este estudio, donde tanto la bebida deportiva como el agua de coco fueron superiores al agua, pero iguales entre sí. 


\section{MHSalud \\ URL www.una.ac.cr/mhsalud}

El hecho de que en éste y otros estudios, tanto la bebida deportiva como el agua de coco resulten más efectivas que el agua para conservar el líquido en el cuerpo (ver la Tabla 3), puede explicarse por el hecho de que ambas bebidas contienen electrolitos y que esto evita un incremento en la diuresis (Maughan et al., 1994). Particularmente, el sodio se encuentra presente en mayor proporción en la bebida deportiva, al compararla con el agua de coco. El sodio ha mostrado ser un electrolito muy importante durante la rehidratación (Maughan, Leiper \& Shirreffs, 1997; Mitchell, Phillips, Mercer, Baylies, \& Pizza, 2000); de hecho, se sabe que su contenido en las bebidas es inversamente proporcional al volumen de orina eliminado y directamente proporcional al porcentaje de líquido conservado (Maughan \& Leiper, 1995; Merson, Maughan \& Shirreffs, 2008). Ésta es probablemente una de las razones por las cuales la bebida deportiva se comportó mejor que el agua.

Por su parte, la explicación para que el agua de coco haya sido tan efectiva a pesar de su bajo contenido de sodio, podría ser su alto contenido de potasio. Se cree que el potasio puede ser tan efectivo como el sodio para conservar el líquido en el cuerpo (Maughan et al., 1994). El potasio podría facilitar la conservación del líquido ingerido luego de hacer ejercicio (Nadel, Mack, \& Nose, 1990, Casa et al., 2005), dado que la deshidratación ocurre tanto en el compartimiento intra como extracelular (Mudambo \& Reynolds, 2001); el potasio podría contribuir a restablecer la pérdida de líquidos en el nivel intracelular (Yawata, 1990).

Tabla 3.

Comparación de los resultados en diferentes estudios. La composición de las bebidas varía de un estudio a otro, sin embargo, las diferencias son pequeñas y su análisis va más allá del alcance de esta discusión.

\begin{tabular}{lllllll}
\hline \multirow{2}{*}{ Autores } & \multicolumn{4}{c}{ BEBIDAS UTILIZADAS } & Indicador \\
\cline { 2 - 5 } & Agua & $\begin{array}{l}\text { Bebida } \\
\text { deportiva }\end{array}$ & $\begin{array}{l}\text { Agua } \\
\text { de coco }\end{array}$ & $\begin{array}{l}\text { Agua de } \\
\text { coco + sodio }\end{array}$ & \\
\hline Madriz-Dávila, 2000 & Menor & Igual & Igual & NE & $\begin{array}{l}\text { \% de reposición de líquido } \\
\text { perdido }\end{array}$ \\
Saat et al., 2002 & Igual & Igual & Igual & NE & \% de rehidratación \\
$\begin{array}{l}\text { Ismail et al., 2007 } \\
\begin{array}{l}\text { Menor } \\
2011\end{array}\end{array}$ & Igual & Igual & Igual & \% de rehidratación \\
\hline
\end{tabular}

NE: No Evaluada

Con respecto a las variables del agrado, se considera que el mayor agrado por la bebida deportiva se podría deber a su contenido de sodio. Algunos estudios han demostrado que la cantidad correcta de sodio puede mejorar la palatabilidad de las bebidas y mejorar su sabor (Passe, Stofan, Rowe, Horswill, \& Murray, 2009). El contenido de sodio tanto en el agua de coco, como en el agua embotellada, es muy bajo.

Por otro lado, según algunos autores, el sabor y en particular el grado de dulzura es uno de los factores principales que afectan el agrado de una bebida, a pesar de existir diferencias en la preferencia del sabor entre las personas y entre las culturas (Meyer, Bar-Or, Salsberg \& Passe, 


\section{MHSalud}

URL www.una.ac.cr/mhsalud

1994, Rivera-Brown, Gutiérrez, Gutiérrez, Frontera, \& Bar-Or, 1999). La diferencia cultural o el gusto de los participantes, pueden explicar la razón por la cual, a pesar de ser el agua embotellada significativamente menos dulce que el agua de coco, no hubo diferencia entre ellas con respecto al agrado del sabor y la aceptación general. De hecho, la dulzura de la bebida en la rehidratación fue a la característica que le dieron menor importancia los participantes de este estudio. Aun considerando lo anterior y sabiendo que, para rehidratar, no se requiere añadir a la bebida componentes energéticos, el hecho de introducir una pequeña cantidad de carbohidratos podría acelerar el transporte de sodio y de agua en el intestino, y mejorar a su vez el sabor de la bebida (Shirreffs \& Maughan, 1997).

En conclusión, tanto el agua de coco como la bebida deportiva son bebidas aptas para la rehidratación post-ejercicio: ambas provocan una menor eliminación de orina y consecuentemente un mayor porcentaje de conservación de líquido al compararlas con el agua. Ambas bebidas muestran una buena tolerancia gastrointestinal en los términos de este estudio. Finalmente, la bebida deportiva presentó mayor aceptación que el agua de coco y el agua embotellada.

Reconocimiento. Este estudio fue financiado por el Gatorade Sports Science Institute ${ }^{\circledR}$, bajo los proyectos de investigación VI-245-A4-303 y VI-245-B0-315 de la Universidad de Costa Rica.

\section{REFERENCIAS}

Aragón-Vargas, L.F. \& Madriz-Dávila, K. (2000). Incomplete warm-climate, post-exercise rehydration with water, coconut water, or a sports drink. Medicine \& Science in Sports and Exercise, 32(5s), 238.

Campbell-Falck. D., Thomas, T., Falck, T.M., Tutuo, N. \& Clem, K. (2000). The intravenous use of coconut water. American Journal of Emergency Medicine, 18(1), 108-11.

Casa, D.J., Clarkson, P.M., \& Roberts, W.O. (2005). American College of Sports Medicine Roundtable on Hydration and Physical Activity: Consensus Statements. Current Sports Medicine Reports, 4(3), 115-127.

Child, R. \& Nathanael, W. (1950). Changes in the sugar composition of coconut water during maturation and germination. Journal of the Science of Food and Agriculture, 1(11), 326329.

Costill, D.L., \& Sparks, K.E. (1973). Rapid fluid replacement after thermal dehydratation. Journal of Applied Physiology, 34, 299-303.

Eiseman, B. (1954). Intravenous infusion of coconut water. AMA Archive of Surgery, 68, 167-78.

Gellish, R.L., Goslin, B.R., Olson, R.E., McDonald, A., Russi, G.D. \& Moudgil, V.K. (2007). Longitudinal Modeling of the Relationship between Age and Maximal Heart Rate. Medicine \& Science in Sports \& Exercise, 39 (5), 822-829.

González-Alonso, J., Heaps, C.L. \& Coyle, E.F. (1992). Rehydration after Exercise with Common Beverages and Water. International Journal of Sports Medicine, 13(5), 399406. 


\section{MHSalud}

URL www.una.ac.cr/mhsalud

Iqbal, Q. (1976). Direct infusion of coconut water. Medical Journal Malaysia, 30(3), 221-223.

Ismail, I., Singh, R. \& Sirisinghe, R.G. (2007). Rehydration with sodium-enriched coconut water after exercise-induced dehydration. The Southeast Asian Journal of Tropical Medicine and Public Health, 38(4), 769-785.

Kuberski, T., Roberts, A., Linehan, B., Bryden, R.N. \& Teburae, M. (1979). Coconut water as a rehydration fluid. New Zealand Medical Journal, 90, 98-100.

Madriz-Dávila, K. (2000). Comparación en la conservación de líquido producida por tres bebidas distintas: agua pura, bebida deportiva y agua de coco, bajo condiciones de deshidratación. Universidad de Costa Rica, Tesis sin publicar (Magister Scientiae). SIBDI: TFG: 19915.

Maughan, R.J. \& Leiper, J.B. (1995). Sodium intake and post-exercise rehydration in man. European Journal of Applied Physiology and Occupational Physiology, 71(4), 311-319.

Maughan, R.J., Leiper, J.B. \& Shirreffs, S.M. (1997). Factors influencing the restoration of fluid and electrolyte balance after exercise in the heat. British Journal of Sports Medicine. 31(3), 175-182. doi:10.1136/bjsm.31.3.175

Maughan, R.J., Owen, J.H., Shirreffs, S.M. \& Leiper, J.B. (1994). Post-Exercise Rehydration In Man: Effects of electrolyte addition to Ingested fluids. European Journal of Applied Physiology and Occupational Physiology, 69, 209-215. doi: 10.1007/BF01094790

Merson, S.J., Maughan, R.J. \& Shirreffs, S.M. (2008). Rehydration with drinks differing in sodium concentration and recovery from moderate exercise-induced hypohydration in man. European Journal of Applied Physiology, 103(5), 585-594. doi: 10.1007/s00421008-0748-0

Meyer, F., Bar-Or, O., Salsberg, A. \& Passe, D. (1994). Hypohydration during exercise in children: effect on thirst, drink preferences \& rehydration. International Journal of Sport Nutrition, 4(1), 22-35.

Mitchell, J.B., Phillips, M.D., Mercer, S.P., Baylies, H.L. \& Pizza, F.X. (2000). Postexercise rehydration: effect of $\mathrm{Na}+$ and volume on restoration of fluid spaces and cardiovascular function. Journal of Applied Physiology, 89, 1302-1309.

Morton, D.P., Aragón-Vargas, L.F. \& Callister, R. (2004). Effect of ingested fluid composition on exercise-related transient abdominal pain. International Journal of Sport Nutrition and Exercise Metabolism, 14(2), 197-208.

Mudambo, S.M. \& Reynolds, N. (2001). Body fluid shifts in soldiers after a jogging/walking exercise in the heat: effects of water and electrolyte solution on rehydration. The Central African Journal of Medicine, 47(9-10), 220-225.

Nadel, E.R., Mack, G.W. \& Nose, H. (1990). Influence of fluid replacement beverages on body fluid homeostasis during exercise and recovery. In: Lamb, D.R. \& Gisolfi, C.V., eds. Perspectives in Exercise Science and Sports Medicine. Fluid Homeostasis During Exercise. Carmel: Benchmark Press, 3, 181-205. 


\section{MHSalud}

URL www.una.ac.cr/mhsalud

Passe, D.H, Stofan, J.R, Rowe, C.L, Horswill, C.A. \& Murray, R. (2009). Exercise condition affects hedonic responses to sodium in a sport drink. Apetite, 52(3), 561-567.

Rivera-Brown, A.M., Gutiérrez, R., Gutiérrez, J.C., Frontera, W.R. \& Bar-Or, O. (1999). Drink composition, voluntary drinking, and fluid balance in exercising, trained, heatacclimatized boys. Journal of Applied Physiology, 86(1), 78-84.

Rolle, R.S. (2007). Good Practice for the Small-scale Production on Bottled Coconut Water. Agricultural and Food Engineering Training and Resource Materials. [Food and Agriculture Organization of the United Nations-FAO]. Recuperado de http://www.fao.org/docrep/010/a1418e/a1418e00.htm, el 20/03/2010.

Saat, M., Singh, R., Sirisinghe, R.G. \& Nawawi, M. (2002). Rehydration after Exercise with Fresh Young Coconut Water, Carbohydrate-Electrolyte Beverage and Plain Water. Journal of Physiological Anthropology and Applied Human Science, 21(2), 93-104.

Sawka, M., Burke, L., Eichner, R., Maughan, R., Montain, S. \& Stachenfeld, N. (2007). American College of Sports Medicine Position Stand: Exercise and Fluid Replacement. Medicine \& Science in Sports \& Exercise, 39(2), 377-390.

Shafiee, M.A., Charest, A.F., Cheema-Dhadli, S., Glick, D.N., Napolova, O., Roozbeh, J.,...Halperin, M.L. (2005). Defining conditions that lead to the retention of water: The importance of the arterial sodium concentration. Kidney International, 67(2), 613-621.

Shirreffs, S.M., Armstrong, L.E. \& Cheuvront, S.N. (2004). Fluid and electrolyte needs for preparation and recovery from training and competition. Journal of Sports Sciences, 22(1), 57-63.

Shirreffs, S.M., \& Maughan, R.J. (1997). Restoration of fluid balance after exercise-induced dehydration: effects of alcohol consumption. Journal of Applied Physiology, 83(4), 11521158 .

Shirreffs, S.M. \& Maughan, R.J. (1998). Volumen repletion following exercise-induced volume depletion in man: replacement of water and sodium losses. American Journal of Physiology, 274(5), 868-875.

Soto-Pradera, E., Fernández, E. \& Calderin, O. (1942). Coconut water: A clinical and experimental study. American Journal of Diseases of Children, 64(6), 977-995.

Vigliar, R., Sdepanian, V. \& Fagundes-Neto, U. (2006). Biochemical profile of coconut water from coconut palms planted in an inland region. Jornal de Pediatría, 82(4), 308-12.

World Health Organization. (2006). Oral rehydration salts production of the new ORS. Geneva, Switzerland. Pages: 123 [http://whqlibdoc.who.int/hq/2006/WHO_FCH_CAH_06.1.pdf ]. Recuperado el 15/05/2010. 
URL www.una.ac.cr/mhsalud

Yawata, T. (1990). Effect of potassium solution on rehydration in rats: comparison with sodium solution and water. The Japanese Journal of Physiology, 40(3), 369-381.

Fecha de recepción: 23 de marzo del 2011.

Fecha de aceptación: 14 de julio del 2011.

Fecha de publicación: 31 de julio del 2011. 